\title{
Solving Qualitative Constraints Involving Landmarks ^
}

\author{
Weiming $\mathrm{Liu}^{1}$, Shengsheng Wang ${ }^{2}$, Sanjiang $\mathrm{Li}^{1}$, and Dayou $\mathrm{Liu}^{2}$ \\ ${ }^{1}$ Centre for Quantum Computation and Intelligent Systems, Faculty of Engineering and \\ Information Technology, University of Technology Sydney, Australia \\ 2 College of Computer Science and Technology, Jilin University, Changchun, China
}

\begin{abstract}
Consistency checking plays a central role in qualitative spatial and temporal reasoning. Given a set of variables $V$, and a set of constraints $\Gamma$ taken from a qualitative calculus (e.g. the Interval Algebra (IA) or RCC-8), the aim is to decide if $\Gamma$ is consistent. The consistency problem has been investigated extensively in the literature. Practical applications e.g. urban planning often impose, in addition to those between undetermined entities (variables), constraints between determined entities (constants or landmarks) and variables. This paper introduces this as a new class of qualitative constraint satisfaction problems, and investigates the new consistency problem in several well-known qualitative calculi, e.g. IA, RCC-5, and RCC-8. We show that the usual local consistency checking algorithm works for IA but fails in RCC-5 and RCC-8. We further show that, if the landmarks are represented by polygons, then the new consistency problem of RCC-5 is tractable but that of RCC-8 is NP-complete.
\end{abstract}

\section{Introduction}

Qualitative constraints are widely used in temporal and spatial reasoning (cf. [1]10|7]). This is partially because they are close to the way humans represent and reason about commonsense knowledge, easy to specify, and provide a flexible way to deal with incomplete knowledge.

Usually, these constraints are taken from a qualitative calculus, which is a set $\mathcal{M}$ of relations defined on an infinite universe $U$ of entities [8]. Well-known qualitative calculi include the Interval Algebra [1], RCC-5 and RCC-8 [10], and the cardinal direction calculus (for point-like objects) [7]. A central problem of reasoning with such a qualitative calculus is the consistency problem. For a qualitative calculus $\mathcal{M}$ on $U$, an instance of the consistency problem over $\mathcal{M}$ is a network $\Gamma$ of constraints like $x \alpha y$, where $x, y$ are variables taken from a finite set $V$, and $\alpha$ is a relation in $\mathcal{M}$. Unlike classical constraint solving, the domain of the variables appeared in a qualitative constraint is usually infinite.

Consistency checking has applications in many areas, e.g. temporal or spatial query preprocessing, planning, natural language understanding; and the consistency problem has been extensively studied for many different qualitative calculi (cf. [3]). These works almost unanimously assume that qualitative constraints involve only unknown entities.

\footnotetext{
* This work was partially supported by an ARC Future Fellowship (FT0990811), an open project program of the MOE Key Lab of Symbolic Computation and Knowledge Engineering (93K17-2009-K03), and by Jilin University Research Project (200903178).
} 
In other words, the precise (geometric) information of every object is totally unknown. In practical applications, however, we often meet constraints that involve both known and unknown entities, i.e. constants and variables.

For example, consider a class scheduling problem in a primary school. In addition to constraints between unknown intervals (e.g. a Math class is followed by a Music class), we may also impose constraints involving determined intervals (e.g. a P.E. class should be during afternoon).

Constraints involving known entities are especially common in spatial reasoning tasks such as urban planning. For example, to find a best location for a landfill, we need to formulate constraints between the unknown landfill and significant landmarks, e.g. lake, university, hospital etc.

In this paper, we explicitly introduce landmarks (defined as known entities) into the definition of the consistency problem, and call the consistency problem involving landmarks the hybrid consistency problem. In comparison, we call the usual consistency problem (involving no landmarks) the pure consistency problem.

In general, solving constraint networks involving landmarks is different from solving constraint networks involving no landmarks. For example, consider the simple RCC-5 algebra. Suppose $x, v_{1}, v_{2}, v_{3}$ are spatial variables which are interpreted as regions in the plane. Consider the following RCC-5 constraint network:

$$
\Gamma=\left\{v_{1} \mathbf{P O} v_{2}, v_{1} \mathbf{P O} v_{3}, v_{2} \mathbf{P O} v_{3}\right\} \cup\left\{x \mathbf{P P} v_{1}, x \mathbf{P P} v_{2}, x \mathbf{P P} v_{3}\right\} .
$$

where $\mathbf{P P}$ is the proper part relation, $\mathbf{P O}$ is the partially overlap relation. It is clear that $\Gamma$ is consistent, and a solution of $\Gamma$ is shown in the following figure (left), where $v_{1}, v_{2}, v_{3}, x$ are interpreted by regions $l_{1}, l_{2}, l_{3}, a$ respectively.
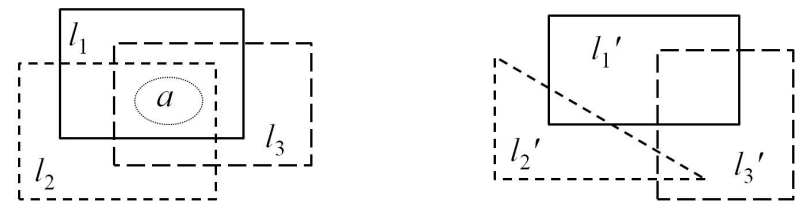

Therefore, the network

$$
\Gamma_{1}=\left\{x \mathbf{P P} l_{1}, x \mathbf{P P} l_{2}, x \mathbf{P P} l_{3}\right\},
$$

which involves three landmarks $l_{1}, l_{2}, l_{3}$, is consistent. Note that the RCC- 5 constraint between any two landmarks is the actual RCC-5 relation between them,

Suppose $l_{1}^{\prime}, l_{2}^{\prime}, l_{3}^{\prime}$ are regions shown in the above figure (right). The network

$$
\Gamma_{2}=\left\{x \mathbf{P P} l_{1}^{\prime}, x \mathbf{P P} l_{2}^{\prime}, x \mathbf{P P} l_{3}^{\prime}\right\}
$$

is not consistent, because $l_{1}^{\prime} \cap l_{2}^{\prime} \cap l_{3}^{\prime}=\varnothing$. The RCC-5 relation between any two of $l_{1}^{\prime}, l_{2}^{\prime}, l_{3}^{\prime}$ is $\mathbf{P O}$, which is the same relation as that between any two landmarks $l_{1}, l_{2}, l_{3}$ in $\Gamma_{1}$. Therefore, the consistency problem for RCC-5 networks involving landmark$\mathrm{s}$ can not be decided by the RCC- 5 relations between the landmarks alone. Note that 
$\left(l_{1}, l_{2}, l_{3}\right)$ and $\left(l_{1}^{\prime}, l_{2}^{\prime}, l_{3}^{\prime}\right)$ are partial solutions of $\Gamma$, so the problem is equivalent to decide whether a particular partial solution can be extended to a global one.

The aim of this paper is to investigate how landmarks affect the consistency of constraint networks in several very important qualitative calculi. The rest of this paper proceeds as follows. Section 2 introduces basic notions in qualitative constraint solving and examples of qualitative calculi. The new consistency problem, as well as several basic results, is also presented here. Assuming that all landmarks are represented as polygons, Section 3 then provides a polynomial decision procedure for the consistency of hybrid basic RCC-5 networks. Besides, if the network is consistent, a solution is constructed in polynomial time; Section 4 shows that consistency problem for hybrid basic RCC-8 networks is NP-hard. The last section then concludes the paper.

\section{Qualitative Calculi and The Consistency Problem}

Most qualitative approaches to spatial and temporal knowledge representation and reasoning are based on qualitative calculi. Suppose $U$ is a universe of spatial or temporal entities. Write $\operatorname{Rel}(U)$ for the algebra of binary relations on $U$. A qualitative calculus on $U$ is a sub-Boolean algebra of $\operatorname{Rel}(U)$ generated by a set $\mathcal{B}$ of jointly exhaustive and pairwise disjoint (JEPD) relations on $U$. Relations in $\mathcal{B}$ are called basic relations of the qualitative calculus. We next recall the well-known Interval Algebra (IA) [1] and the two RCC algebras.

Example 1 (Interval Algebra). Let $U$ be the set of closed intervals on the real line. Thirteen binary relations between two intervals $x=\left[x^{-}, x^{+}\right]$and $y=\left[y^{-}, y^{+}\right]$are defined by comparing the order relations between the endpoints of $x$ and $y$. These are the basic relations of IA.

Example 2 (RCC-5 and RCC-8 Algebras ${ }^{3}$ ). Let $U$ be the set of bounded regions in the real plane, where a region is a nonempty regular set. The RCC- 8 algebra is generated by the eight topological relations

$$
\text { DC, EC, PO, EQ, TPP, NTPP, } \mathbf{T P P}^{\sim}, \mathbf{N T P P}^{\sim},
$$

where DC, EC, PO, TPP and NTPP are defined in Table 1, EQ is the identity relation, and $\mathbf{T P P} \mathbf{P}^{\sim}$ and $\mathbf{N T P P} \mathbf{P}^{\sim}$ are the converses of $\mathbf{T P P}$ and $\mathbf{N T P P}$, respectively, see the following figure for illustration. The RCC-5 algebra is the sub-algebra of RCC-8 generated by the five part-whole relations

$$
\mathrm{DR}, \mathrm{PO}, \mathrm{EQ}, \mathrm{PP}, \mathrm{PP}^{\sim},
$$

where $\mathbf{D R}=\mathbf{D C} \cup \mathbf{E C}, \mathbf{P P}=\mathbf{T P P} \cup \mathbf{N T P P}$, and $\mathbf{P P}^{\sim}=\mathbf{T P P} \mathbf{P}^{\sim} \cup \mathbf{N T P} \mathbf{P}^{\sim}$.

A qualitative calculus provides a useful constraint language. Suppose $\mathcal{M}$ is a qualitative calculus defined on an infinite domain $U$. Relations in $\mathcal{M}$ can be used to express

\footnotetext{
${ }^{3}$ We note that the RCC algebras have interpretations in arbitrary topological spaces. In this paper, we only consider the most important interpretation in the real plane.
} 
Table 1. A topological interpretation of basic RCC- 8 relations in the plane, where $a, b$ are two bounded plane regions, and $a^{\circ}, b^{\circ}$ are the interiors of $a, b$, respectively.

\begin{tabular}{c|c|c|c} 
Relation & Meaning & Relation & Meaning \\
\hline DC & $a \cap b=\varnothing$ & TPP & $a \subset b, a \not \subset b^{\circ}$ \\
EC & $a \cap b \neq \varnothing, a^{\circ} \cap b^{\circ}=\varnothing$ & NTPP & $a \subset b^{\circ}$ \\
PO & $a \nsubseteq b, b \nsubseteq a, a^{\circ} \cap b^{\circ} \neq \varnothing$ & EQ & $a=b$
\end{tabular}
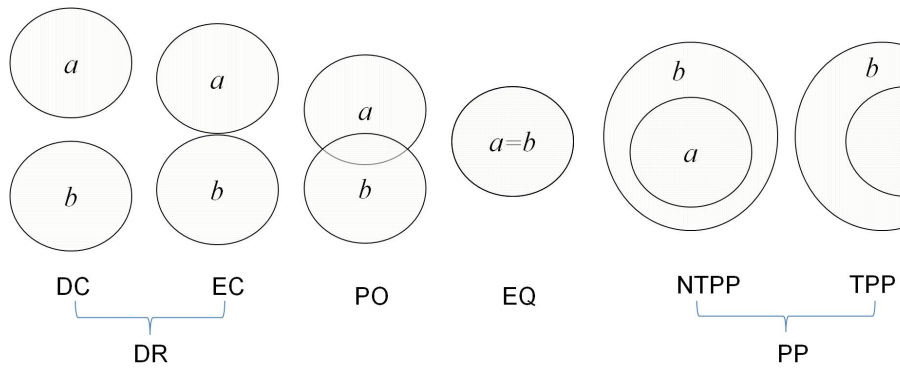

constraints about variables which takes values in $U$. A constraint has the form $x_{1} \alpha x_{2}$, where $\alpha$ is a relation in $\mathcal{M}, x_{i}$ is either a constant in $U$ (called landmark in this paper), or a variable. Such a constraint is basic if $\alpha$ is a basic relation in $\mathcal{M}$.

Given a finite set $\Gamma$ of constraints, we write $V(\Gamma)(L(\Gamma)$, resp.) for the set of variables (constants, resp.) appearing in $\Gamma$, and assume that the constraint between two landmarks $a, b$ is the actual basic relation in $\mathcal{M}$ that relates $a$ to $b$. A solution of $\Gamma$ is an assignment of values in $U$ to variables in $V(\Gamma)$ such that all constraints in $\Gamma$ are satisfied. If $\Gamma$ has a solution, we say $\Gamma$ is consistent or satisfiable. Two sets of constraint $\Gamma$ and $\Gamma^{\prime}$ are equivalent if they have the same set of solutions.

A set $\Gamma$ of constraints is said to be a complete constraint network if there is a unique constraint between each pair of variables/constants appearing in $\Gamma$. It is straightforward to show that a non-complete constraint network $\Gamma$ can be transformed into an equivalent complete constraint network $\Gamma^{\prime}$ in polynomial time.

Definition 1. Let $\mathcal{M}$ be a qualitative calculus on $U$. The hybrid consistency problem of $\mathcal{M}$ is, given a constraint network $\Gamma$ in $\mathcal{M}$, decide the consistency of $\Gamma$ in $\mathcal{M}$, i.e. decide if there is an assignment of elements in $U$ to variables in $\Gamma$ that satisfies all the constraints in $\Gamma$. The pure consistency problem of $\mathcal{M}$ is the sub-consistency problem that considers constraint networks that involve no landmarks.

The hybrid consistency problem of $\mathcal{M}$ can be approximated by a variant of the pathconsistency algorithm. We say a complete constraint network $\Gamma$ is path-consistent if for any three objects $l_{i}, l_{j}, l_{k}$ in $V(\Gamma) \cup L(\Gamma)$, we have

$$
\alpha_{i j}=\alpha_{j i}^{\sim} \& \alpha_{i j} \subseteq \alpha_{i k} \circ_{w} \alpha_{k j}
$$

where $\circ_{w}$ is the weak composition [6]8] in $\mathcal{M}$ and $\alpha{ }^{\circ} \beta$ is defined to be the smallest relation in $\mathcal{M}$ which contains the usual composition of $\alpha$ and $\beta$, i.e.

$$
\alpha \circ_{w} \beta=\bigcup\{\gamma \text { is a basic relation in } \mathcal{M}: \gamma \cap \alpha \circ \beta \neq \varnothing\} \text {. }
$$


We note that the above notion of path-consistency for qualitative constraint network is very different from the classical notion (cf. [5]3]).

It is clear that each complete network can be transformed in polynomial time into an equivalent complete network that is path-consistent. Because the consistency problem is in general NP-hard, we do not expect that a local consistency algorithm can solve the general consistency problem. However, it has been proved that the path-consistency algorithm suffices to decide the pure consistency problem for large fragments of some well-known qualitative calculi, e.g. IA, RCC-5, and RCC-8 (cf. [3]). This shows that, at least for these calculi, the pure consistency problem can be solved by path-consistency algorithm and by applying the backtracking method to constraints [3].

The remainder of this paper will investigate the hybrid consistency problem for the above calculi. In the following discussion, we assume $\Gamma$ is a complete basic network that involves at least one landmark.

For IA, we have

Proposition 1. Suppose $\Gamma$ is a basic network of IA constraints that involves landmarks and variables. Then $\Gamma$ is consistent iff it is path-consistent.

Proof. If we replace each landmark in $\Gamma$ by a new interval variable, and constrain any two new variables with the actual relation between the corresponding landmarks, then we obtain a basic network $\Gamma^{*}$ of IA constraints that involves no landmarks. Note that each path-consistent IA basic network is globally consistent. The landmarks (as a partial solution of $\Gamma^{*}$ ) can also be extended to a solution.

This result shows that, for IA, the hybrid consistency problem can be solved in the same way as the pure consistency problem. Similar conclusion also holds for some other calculi, e.g. the Point Algebra, the Rectangle Algebra, and the Cardinal Direction Calculus (for point-like objects) [7].

This property, however, does not hold in general. Take the RCC-5 as example. If a basic network $\Gamma$ involves no landmark, then we know $\Gamma$ is consistent if it is pathconsistent. If $\Gamma$ involves landmarks, we have seen in the introduction a path-consistent but inconsistent basic RCC-5 network.

In the next two sections, we investigate how landmarks affect the consistency of RCC-5 and RCC-8 topological constraints. We stress that, in this paper, we only consider the standard (and the most important) interpretation of the RCC language in the real plane, as given in Example 2 When restricting landmarks to polygons, we first show that the consistency of a hybrid basic RCC-5 network can still be decided in polynomial time (Section 4), but that of RCC-8 networks is NP-hard.

\section{The Hybrid Consistency Problem of RCC-5}

We begin with a short review of the realization algorithm for pure consistency problem of RCC-5 [45]. Suppose $\Gamma$ involves only spatial variables $v_{1}, v_{2}, \cdots, v_{n}$. We define a finite set $X_{i}$ of control points for each $v_{i}$ as follows:

- Add a point $P_{i}$ to $X_{i}$;

- For any $j>i$, add a new point $P_{i j}$ to both $X_{i}$ and $X_{j}$ if $\left(v_{i} \mathbf{P O} v_{j}\right) \in \Gamma$; 
- For any $j$, put all points in $X_{i}$ into $X_{j}$ if $\left(v_{i} \mathbf{P P} v_{j}\right) \in \Gamma$.

Take $\varepsilon>0$ such that the distance between any two different points in $\bigcup_{i=1}^{n} X_{i}$ is greater than $2 \varepsilon$. Let $B(P, \varepsilon)$ be the closed disk with radius $\varepsilon$ centred at $P$. By the choice of $\varepsilon$, different disks are disjoint. Let $a_{i}=\bigcup\left\{B(P, \varepsilon): P \in X_{i}\right\}$. It is easy to check that the assignment is a solution of $\Gamma$, if $\Gamma$ is consistent.

Assume $\Gamma$ is a basic RCC-5 network involving landmarks $L=\left\{l_{1}, \cdots, l_{m}\right\}$ in the real plane and variables $V=\left\{v_{1}, \cdots, v_{n}\right\}$. Write $\partial L$ for the union of the boundaries of the landmarks. A block is defined to be a maximal connected component of $\mathbb{R}^{2} \backslash \partial L$, which is an open set. It is clear that the complement of the union of all landmarks (which are bounded) is the unique unbounded block. We write $\mathbb{B}$ for the set of all blocks.

For each landmark $l_{i}$, we write $I\left(l_{i}\right)$ for the set of blocks that $l_{i}$ contains, and write $E\left(l_{i}\right)$ for the set of rest blocks, i.e. the blocks that are disjoint from $l_{i}$. That is,

$$
I\left(l_{i}\right)=\left\{b \in \mathbb{B}: b \subseteq l_{i}\right\}, \quad E\left(l_{i}\right)=\left\{b \in \mathbb{B}: b \cap l_{i}=\varnothing\right\} .
$$

It is clear that each block is in either $I\left(l_{i}\right)$ or $E\left(l_{i}\right)$, but not both, i.e., $I\left(l_{i}\right) \cup E\left(l_{i}\right)=\mathbb{B}$ and $I\left(l_{i}\right) \cap E\left(l_{i}\right)=\varnothing$.

These constructions can be extended from landmarks to variables as

$$
\begin{aligned}
I\left(v_{i}\right) & =\bigcup\left\{I\left(l_{j}\right): l_{j} \mathbf{P} \mathbf{P} v_{i}\right\}, \\
E\left(v_{i}\right) & =\bigcup\left\{I\left(l_{j}\right): l_{j} \mathbf{D R} v_{i}\right\} \cup \bigcup\left\{E\left(l_{j}\right): v_{i} \mathbf{P} \mathbf{P} l_{j}\right\} .
\end{aligned}
$$

Intuitively, $I\left(v_{i}\right)$ is the set of blocks that $v_{i}$ must contain, and $E\left(v_{i}\right)$ is the set of blocks that should be excluded from $v_{i}$. We now give an example.

Example 3. Consider the network $\Gamma_{1}$ that involves landmarks $l_{1}, l_{2}, l_{3}$ and variable $v$, where $l_{2} \mathbf{D R} l_{3}$ and $l_{1}=l_{2} \cup l_{3}$ (see the following figure). The constraints in $\Gamma_{1}$ are specified as $v \mathbf{P P} l_{1}, v \mathbf{D R} l_{2}$ and $v \mathbf{D R} l_{3}$. We have $\mathbb{B}=\left\{b_{1}, b_{2}, b_{3}\right\}$, and

$$
\begin{array}{lll}
I\left(l_{1}\right)=\left\{b_{1}, b_{2}\right\}, & I\left(l_{2}\right)=\left\{b_{1}\right\}, & I\left(l_{3}\right)=\left\{b_{2}\right\}, \\
E\left(l_{1}\right)=\left\{b_{3}\right\}, & E\left(l_{2}\right)=\left\{b_{2}, b_{3}\right\}, & E\left(l_{3}\right)=\left\{b_{1}, b_{3}\right\}, \\
I(v)=\varnothing & E(v)=E\left(l_{1}\right) \cup I\left(l_{2}\right) \cup I\left(l_{3}\right)=\mathbb{B} . &
\end{array}
$$

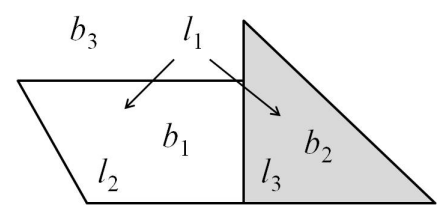

The following proposition claims that no block can appear in both $I\left(v_{i}\right)$ and $E\left(v_{i}\right)$.

Proposition 2. Suppose $\Gamma$ is a basic RCC-5 constraint network that involves at least one landmark. If $\Gamma$ is path-consistent, then $I\left(v_{i}\right) \cap E\left(v_{i}\right)=\varnothing$. 
Proof. Assume $b \in I\left(v_{i}\right) \cap E\left(v_{i}\right)$. There exists some $l_{j}$ such that $l_{j} \mathbf{P P} v_{i}$ and $b \in I\left(l_{j}\right)$. Furthermore, there exists some $l_{k}$ such that either (i) $l_{k} \mathbf{D R} v_{i}$ and $b \in I\left(l_{k}\right)$, or (ii) $v_{i} \mathbf{P P} l_{k}$ and $b \in E\left(l_{k}\right)$.

Both cases lead to contradiction. For the first case, we know that $b \subseteq l_{j} \cap l_{k}$, while the path-consistency of $\Gamma$ implies that $l_{j} \mathbf{D R} l_{k}$. For the second case, the pathconsistency of $\Gamma$ implies $l_{j} \mathbf{P P} l_{k}$, but $b \subseteq l_{j}$ and $b \cap l_{k}=\varnothing$.

We have the following theorem.

Theorem 1. Suppose $\Gamma$ is a basic RCC-5 constraint network that involves at least one landmark. If $\Gamma$ is consistent, then we have

- For any $v_{i} \in V$,

$$
E\left(v_{i}\right) \subsetneq \mathbb{B} .
$$

- For any $v_{i} \in V$ and $w \in L \cup V$ such that $\left(v_{i} \mathbf{P O} w\right) \in \Gamma$,

$$
\begin{gathered}
E\left(v_{i}\right) \cup E(w) \subsetneq \mathbb{B}, \\
E\left(v_{i}\right) \cup I(w) \subsetneq \mathbb{B}, \\
I\left(v_{i}\right) \cup E(w) \subsetneq \mathbb{B} .
\end{gathered}
$$

- For any $v_{i} \in V$ and $l_{j} \in L$ such that $\left(v_{i} \mathbf{P P} l_{j}\right) \in \Gamma$,

$$
I\left(v_{i}\right) \subsetneq I\left(l_{j}\right) .
$$

- For any $v_{i} \in V$ and $l_{j} \in L$ such that $\left(l_{j} \mathbf{P P} v_{i}\right) \in \Gamma$,

$$
E\left(v_{i}\right) \subsetneq E\left(l_{j}\right) .
$$

- For any $v_{i}, v_{j} \in V$ such that $\left(v_{i} \mathbf{P} \mathbf{P} v_{j}\right) \in \Gamma$,

$$
I\left(v_{i}\right) \cup E\left(v_{j}\right) \subsetneq \mathbb{B} .
$$

Proof. Note the inclusion part of these equations are clear. We only focus on the inequality. Suppose $\left\{\bar{v}_{1}, \cdots, \bar{v}_{n}\right\}$ is a solution of $\Gamma$. Because each $\bar{v}_{i}$ has nonempty interior, there exists at least one block $b$ such that $b \cap \bar{v}_{i}$ is nonempty. Clearly, $b \notin E\left(v_{i}\right)$ since blocks in $E\left(v_{i}\right)$ are all disjoint from $\bar{v}_{i}$. Therefore, $E\left(v_{i}\right) \neq \mathbb{B}$.

If $\left(v_{i} \mathbf{P O} w\right) \in \Gamma$, then by assumption we have $\bar{v}_{i} \mathbf{P O} \bar{w}$, where $\bar{w}$ is $l_{j}$ if $w=l_{j}$. By definition of $\mathbf{P O}$ (see Table 1 ), we know $\bar{v}_{i}$ and $\bar{w}$ have a common interior point. This implies that there exists a block $b$ that contains an interior point of $\bar{v}_{i} \cap \bar{w}$. This block is neither in $E\left(v_{i}\right)$ nor in $E(w)$. That is, $E\left(v_{i}\right) \cup E(w) \neq \mathbb{B}$. Similarly, we know neither $E\left(v_{i}\right) \cup I(w)$ nor $I\left(v_{i}\right) \cup E(w)$ is $\mathbb{B}$. If $\left(v_{i} \mathbf{P} \mathbf{P} l_{j}\right) \in \Gamma$, then $\bar{v}_{i} \mathbf{P P} l_{j}$. Because $l_{j}$ is the regularized union of all the blocks it contains, we know there exists at least one block in $I\left(l_{j}\right)$ that is not in $I\left(v_{i}\right)$. This shows $I\left(v_{i}\right) \neq I\left(l_{j}\right)$. The rest situations are similar.

These conditions are also sufficient to determine the consistency of a path-consistent basic RCC-5 network. We show this by devising a realization algorithm. The construction is similar to that for the pure consistency problem. For each $v_{i}$, we define a finite set $X_{i}$ of control points as follows, where for clarity, we write

$$
P\left(v_{i}\right)=\mathbb{B}-I\left(v_{i}\right)-E\left(v_{i}\right) .
$$


- For each block $b$ in $P\left(v_{i}\right)$, select a fresh point in $b$ and add the point into $X_{i}$.

- For any $j>i$ with $\left(v_{i} \mathbf{P O} v_{j}\right) \in \Gamma$, select a fresh point in some block $b$ in $P\left(v_{i}\right) \cap$ $P\left(v_{j}\right)$ (if it is not empty), and add the point into $X_{i}$ and $X_{j}$.

- For any $j$, put all points in $X_{j}$ into $X_{i}$ if $\left(v_{j} \mathbf{P P} v_{i}\right) \in \Gamma$.

We note that the points selected from a block $b$ for different $v_{i}$, or in different steps, should be pairwise different. Recall that each point in $\bigcup_{i=1}^{n} X_{i}$ is not at the boundary of any block. We choose $\varepsilon>0$ such that $B(P, \varepsilon)$ does not intersect either the boundary of a block or another disk $B(Q, \varepsilon)$. Furthermore, we can assume that $\varepsilon$ is small enough such that the union of all the disks $B(P, \varepsilon)$ does not cover any block in $\mathbb{B}$.

Let

$$
\hat{a}_{i}=\bigcup\left\{B(P, \varepsilon): P \in X_{i}\right\} \cup \bigcup\left\{l_{j}: l_{j} \mathbf{P P} v_{i}\right\} .
$$

We claim that $\left\{\hat{a}_{1}, \cdots, \hat{a}_{t}\right\}$ is a solution of $\Gamma$. We first prove the following lemma.

Lemma 1. Let $\Gamma$ be a path-consistent basic RCC-5 constraint network that involves at least one landmark. Suppose $\mathbb{B}$ is the block set of $\Gamma$. Then, for each $b \in \mathbb{B}$, we have

$-b \in I\left(v_{i}\right)$ iff $b \subseteq \hat{a}_{i}$.

$-b \in E\left(v_{i}\right)$ iff $b \cap \hat{a}_{i}=\varnothing$.

$-b \in P\left(v_{i}\right)$ iff $b \nsubseteq \hat{a}_{i}$ and $b \cap \hat{a}_{i} \neq \varnothing$.

Proof. We first prove the necessity part.

Suppose $b \in I\left(v_{i}\right)$. There exists a landmark $l$ such that $l \mathbf{P} \mathbf{P} v_{i}$ and $b \subseteq l$. The first statement follows directly from $b \subseteq l$ and $l \subseteq \hat{a}_{i}$.

Assume $b \in E\left(v_{i}\right)$. By definition, there is a landmark $l$ such that either (i) $b \subseteq l$ and $l \mathbf{D R} v_{i}$ or (ii) $b \cap l=\varnothing$ and $v_{i} \mathbf{P P} l$. In both cases, we have $b \cap l^{\prime}=\varnothing$ for any landmark $l^{\prime}$ with $l^{\prime} \mathbf{P P} v_{i}$. We next show $b \cap B(P, \varepsilon)=\varnothing$ for any $P$ in $X_{i}$, which is equivalent to that there is no control point in $X_{i}$ in $b$. Now suppose $P$ is a control point in $X_{i}$ and $P \in b$. Since $b \in E\left(v_{i}\right)$, we know $P$ is not generated by the first two rules. That is, $P$ must be a control point of some $v_{j}$ and $v_{j} \mathbf{P P} v_{i}$. In this case, it can be proved that $b \in E(v)$ by path-consistency. Therefore we find a different variable $v_{j}$ such that $b \in E\left(v_{j}\right)$ and $b \cap \hat{a}_{j} \neq \varnothing$. Because the variables are finite, we will get a contradiction by repeating this procedure. As a conclusion, we have $b \cap \hat{a}_{i}=\varnothing$ whenever $b \in E\left(v_{i}\right)$.

Now assume $b \in P\left(v_{j}\right)$. The first step of the construction algorithm shows that a control point of $v_{j}$ is taken from $b$. Therefore, $b \cap \hat{a}_{j} \neq \varnothing$. Since $b \notin I\left(v_{j}\right)$, we know $b$ is not contained in any landmark $l$ with $l \mathbf{P P} v_{i}$. Moreover, $b$ is not contained in the union of all $B(P, \varepsilon)$ due to the choice of $\varepsilon$. This implies $b \not \hat{a}_{i}$.

Since $\left\{I\left(v_{i}\right), E\left(v_{i}\right), P\left(v_{i}\right)\right\}$ is a partition of the blocks in $B$, it is easy to see the conditions are also sufficient.

We next prove that $\left\{\hat{a}_{1}, \cdots, \hat{a}_{t}\right\}$ is a solution of $\Gamma$.

Theorem 2. Suppose $\Gamma$ is a complete basic RCC-5 network involving landmarks $L$ and variables $V$. Assume $\Gamma$ is path-consistent and satisfies the conditions in Theorem 1 . Then $\Gamma$ is consistent and $\left\{\hat{a}_{1}, \cdots, \hat{a}_{t}\right\}$, as constructed in $(16)$, is a solution of $\Gamma$. 
Proof. By (8) we know there is at least one block $b$ in either $I\left(v_{i}\right) \cup P\left(v_{i}\right)$. By Lemma 1 we know $\hat{a}_{i}$ is nonempty. We next prove all constraints in $\Gamma$ are satisfied.

We first consider the constraint $v_{i} \alpha l_{j}$ between variable $v_{i}$ and landmark $l_{j}$. The cases that $\alpha=\mathbf{P P}, \mathbf{P P}^{\sim}, \mathbf{D R}$ can be directly checked by Lemma 1. Now suppose $v_{i} \mathbf{P O} l_{j}$. By $(9)$, we know that $E\left(v_{i}\right) \cup E\left(l_{j}\right) \subsetneq \mathbb{B}$. That is, there is some block $b$ in $I\left(l_{j}\right)$ but outside $E\left(v_{i}\right)$. By Lemma 1, we know $b \cap \hat{a}_{i} \neq \varnothing$. By $b \subseteq l_{j}, \hat{a}_{i}$ and $l_{j}$ have a common interior point. Furthermore, by $\left.E\left(v_{i}\right) \cup I\left(l_{j}\right) \subsetneq \mathbb{B} \sqrt{10}\right)$, we know there is a block $b^{\prime}$ in $E\left(l_{j}\right)$ that is outside $E\left(v_{i}\right)$. By $b^{\prime} \in E\left(l_{j}\right)$, we have $b^{\prime} \cap l_{j}=\varnothing$; by $b^{\prime} \notin E\left(v_{i}\right)$ and Lemma 1, we have $b^{\prime} \cap \hat{a}_{i} \neq \varnothing$. So $\hat{a}_{i} \nsubseteq l_{j}$. Similarly, we can show $l_{j} \nsubseteq \hat{a}_{i}$. Therefore, $\hat{a}_{i} \mathbf{P O} l_{j}$.

Now we consider constraints between two variables $v_{i}$ and $v_{j}$.

(1) If $\left(v_{i} \mathbf{P P} v_{j}\right) \in \Gamma$, we have $X_{i} \subset X_{j}$ and $I\left(v_{i}\right) \subseteq I\left(v_{j}\right)$. By definition, $\hat{a}_{i} \subseteq \hat{a}_{j}$. Moreover, by $I\left(v_{i}\right) \cup E\left(v_{j}\right) \subsetneq \mathbb{B}(14)$, we know there is a block $b$ that is outside both $I\left(v_{i}\right)$ and $E\left(v_{j}\right)$. By Lemma 1 this implies that $b \nsubseteq \hat{a}_{i}$ and $b \cap \hat{a}_{j} \neq \varnothing$. If $b \cap \hat{a}_{i}=\varnothing$ or $b \subseteq \hat{a}_{j}$, then $\hat{a}_{i} \neq \hat{a}_{j}$. If otherwise, then $b \in P\left(v_{j}\right)$. Hence, there is a fresh control point $P$ of $v_{j}$ in $b$. By the choice of $P$, we know $P$ is not in $X_{i}$, hence not in $\hat{a}_{i}$. So in this case we also have $\hat{a}_{i} \neq \hat{a}_{j}$. Therefore, we have $\hat{a}_{i} \mathbf{P P} \hat{a}_{j}$.

(2) If $\left(v_{i} \mathbf{P} \mathbf{P}^{\sim} v_{j}\right) \in \Gamma$, we know that $\Gamma$ also contains constraint $\left(v_{j} \mathbf{P P} v_{i}\right)$. Because we have proved that $\hat{a}_{j} \mathbf{P} \mathbf{P} \hat{a}_{i}$, constraint $v_{i} \mathbf{P} \mathbf{P} \sim v_{j}$ is also satisfied by $\hat{a}_{i}$ and $\hat{a}_{j}$.

(3) If $\left(v_{i} \mathbf{D R} v_{j}\right) \in \Gamma$, we show that $X_{i} \cap X_{j}=\varnothing$. Otherwise, there exists some $v_{k}$ such that $v_{k} \mathbf{P P} v_{i}$ and $v_{k} \mathbf{P P} v_{j}$, which contradicts $v_{i} \mathbf{D R} v_{j}$ by path-consistency. It remains to prove $X_{i} \cap l=\varnothing$ if $\left(l \mathbf{P P} v_{j}\right) \in \Gamma$, and $X_{j} \cap l^{\prime}=\varnothing$ if $\left(l^{\prime} \mathbf{P} \mathbf{P} v_{i}\right) \in \Gamma$.

Let $P$ be a control point of $v_{i}$, and $l$ is a landmark such that $l \mathbf{P} \mathbf{P} v_{j}$. We next show $P \notin l$. By $v_{i} \mathbf{D R} v_{j}$ and $l \mathbf{P P} v_{j}$, we know $l \mathbf{D R} v_{i}$. Hence $E(l) \subseteq E\left(v_{i}\right)$. For any block $b \in E(l)$, by $b \in E\left(v_{i}\right)$ and Lemma 1, we know $b \cap \hat{a}_{i}=\varnothing$. Because $P \in \hat{a}_{i}$, we know $P \notin b$ for any $b \in E(l)$. This implies that $P \notin l$. Therefore, $X_{i} \cap l=\varnothing$ if $l \mathbf{P P} v_{j}$. That $X_{j} \cap l^{\prime}=\varnothing$ if $l^{\prime} \mathbf{P} \mathbf{P} v_{j}$ is similar. In conclusion, we have $\hat{a}_{i} \mathbf{D R} \hat{a}_{j}$.

(4) If $\left(v_{i} \mathbf{P O} v_{j}\right) \in \Gamma$, we show $\hat{a}_{i}$ and $\hat{a_{j}}$ have a common interior point. We prove this by contradiction. Suppose $v_{i} \mathbf{P O} v_{j}$ but $\hat{a}_{i}$ and $\hat{a}_{j}$ have no common interior point. For any $b \in I\left(v_{i}\right)$, we have $b \subseteq \hat{a}_{i}$. Since $b$ is an open set, $b \cap \hat{a}_{j}$ cannot be nonempty (otherwise $\hat{a}_{i}$ and $\hat{a}_{j}$ shall have a common interior point). Therefore $b \in E\left(v_{j}\right)$, according to Lemma 1 . In other words, $I\left(v_{i}\right) \subseteq E\left(v_{j}\right)$. Symmetrically, we have $I\left(v_{j}\right) \subseteq E\left(v_{i}\right)$. Hence $I\left(v_{i}\right) \cup I\left(v_{j}\right) \cup E\left(v_{i}\right) \cup E\left(v_{j}\right)=E\left(v_{i}\right) \cup E\left(v_{j}\right)$. Note the right hand side of the above equation is a proper subset of $\mathbb{B}$ (cf. (9p)). This implies that $P\left(v_{i}\right) \cap P\left(v_{j}\right) \neq \varnothing$. By the construction of control points, we know there exists $P \in X_{i} \cap X_{j}$, where $P$ is a control point selected from a block in $P\left(v_{i}\right) \cap P\left(v_{j}\right)$. Because $P$ is a common interior point of both $\hat{a}_{i}$ and $\hat{a}_{j}$, this clearly contradicts our assumption. Therefore, $\hat{a}_{i}$ and $\hat{a}_{j}$ have a common interior point. That $\hat{a}_{i}$ and $\hat{a}_{j}$ are incomparable is similar to the case of $\left(v_{i} \mathbf{P O} l_{j}\right)$. As a result, we know $\hat{a}_{i} \mathbf{P O} \hat{a}_{j}$.

In summary, all constraints are satisfied and $\left\{\hat{a}_{1}, \cdots, \hat{a}_{t}\right\}$ is a solution of $\Gamma$.

It is worth noting that the complexity of deciding the consistency of a hybrid basic RCC-5 network includes two parts, viz. the complexity of computing the blocks, and that of checking the conditions in Theorem 1. The latter part alone can be completed in $O(|\mathbb{B}| n(n+m))$ time, where $|\mathbb{B}|$ is the number of the blocks. In the worst situation, the number of blocks may be up to $2^{m}$. This suggests that the decision method described 
above is in general inefficient. The following theorem, however, asserts that this method is still polynomial in the size of the input instance, provided that the landmarks are all represented as polygons.

Before proving Theorem 3, we review some notions and results in computational geometry. The reader is referred to [2] and references therein for more details. A (planar) subdivision is the map induced by a planar embedding of a graph. The embedding of nodes (arcs, resp.) of the graph is called vertices (edges, resp.) in the subdivision, where each edge is required to be a straight line segment. A face of the subdivision is a maximal connected subset of the remaining part of the plane excluded by all the edges and vertices. The complexity of a subdivision is defined to be the sum of the number of vertices, the number of edges, and the number of faces in the subdivision. The overlay of two subdivisions $S_{1}$ and $S_{2}$ is the subdivision of the plane induced by all the edges from $S_{1}$ and $S_{2}$. Let $S_{1}$ and $S_{2}$ be two subdivisions with complexities $n_{1}$ and $n_{2}$. The overlay of $S_{1}$ and $S_{2}$ can be computed in $O(n \log n+k \log n)$ time, where $n=n_{1}+n_{2}$ and $k$ is the complexity of the overlay [2, Section 2.3]. Note that this complexity is sensitive to the output. Polygons can be viewed as special cases of subdivisions.

Theorem 3. Suppose $\Gamma$ is a basic RCC-5 constraint network, and $V(\Gamma)=\left\{v_{1}, \cdots, v_{n}\right\}$ and $L(\Gamma)=\left\{l_{1}, \cdots, l_{m}\right\}$ are the set of variables and, respectively, the set of landmark$s$ appearing in $\Gamma$. Assume each landmark $l_{i}$ is represented by a planar subdivision with complexity $k_{i}$. Let $K$ be the sum of all $k_{i}$. Then the consistency of $\Gamma$ can be decided in $O\left((n+m)^{3}+n(n+m) K^{2}+m^{2} K^{2} \log K\right)$ time.

Proof. We first compute the overlay of all landmarks. Then we calculate $I\left(l_{i}\right)$ and $E\left(l_{i}\right)$ for each landmark $\left(l_{i}\right)$, and $I\left(v_{i}\right)$ and $E\left(v_{i}\right)$ for each variable $v_{i}$. Finally we check the conditions listed in Theorem 1

Let $\mathcal{O}_{k}$ be the overlay of the first $k$ landmarks, and write $\mathcal{O}=\mathcal{O}_{m}$. Recall each overlay is a subdivision. We show that the complexity of $\mathcal{O}$ is $O\left(K^{2}\right)$. Each vertex in the subdivision $\mathcal{O}$ is either a vertex of some landmark, or the intersection of two edges of the landmarks. Because the total number of vertices (edges, resp.) is less than $K$, we have that the number of vertices in $\mathcal{O}$ is $O\left(K^{2}\right)$. Each edge in $\mathcal{O}$ is clearly a part of an edge of some landmark. Moreover, each edge in a landmark is divided into at most $K$ edges in $\mathcal{O}$, so the number of edges in $\mathcal{O}$ is $O\left(K^{2}\right)$. Let $l_{i}^{\prime}$ be the subdivision obtained by replacing the line segments in $l_{i}$ with lines. ${ }^{4}$ It is obvious that the overlay $\mathcal{O}^{\prime}$ of all $l_{i}^{\prime}$ is finer than $\mathcal{O}$. Because $K$ lines partition the plane into at most $1+1+2+\cdots+K=O\left(K^{2}\right)$ faces, we know that the number of faces in $\mathcal{O}^{\prime}$ is $O\left(K^{2}\right)$, which further implies that the number of faces in $\mathcal{O}$ is also $O\left(K^{2}\right)$. In summary, the complexity of subdivision $\mathcal{O}$ is $O\left(K^{2}\right)$. It is clear that the faces in $\mathcal{O}$ are actually the blocks we defined.

Now consider how to compute subdivision $\mathcal{O}_{i+1}$, the overlay of subdivision $\mathcal{O}_{i}$ and landmark $l_{i+1}$. Regarded as a subdivision, the complexity of $l_{i+1}$ is $O(K)$. The complexities of $\mathcal{O}_{k}$ and $\mathcal{O}_{i+1}$ are no more than that of $\mathcal{O}$, which is $O\left(K^{2}\right)$. By the computational geometry result stated before the theorem, the subdivision $\mathcal{O}_{i+1}$ can be computed in $O\left(K^{2} \log K\right)$ time. Therefore, the overlay $\mathcal{O}$ of all the landmarks can be computed in $O\left(m K^{2} \log K\right)$ time.

\footnotetext{
${ }^{4}$ Note here we allow the edges in a subdivision to be rays.
} 
To record whether a face is contained in a landmark or not, we attach to each face $f$ (in some overlay $\mathcal{O}_{i}$ ) a label which is the set of landmarks that contain face $f$. When computing the overlay $\mathcal{O}_{i+1}$ of $\mathcal{O}_{i}$ and $l_{i+1}$, the labels of faces in $\mathcal{O}_{i+1}$ can be computed as well. This is because, each face in $\mathcal{O}_{i+1}$ is the intersection of some face $f_{1}$ from $\mathcal{O}_{i}$ and some face $f_{2}$ from $l_{i+1}$, and its label is the union of the labels of $f_{1}$ and $f_{2}$, which can be computed in $O(m)$ time. Computing the labels of faces increases the complexity of calculating the subdivision $\mathcal{O}$ to $O\left(m^{2} K^{2} \log K\right)$ time.

For each landmark $l_{i}, I\left(l_{i}\right)$ is the set of faces in $\mathcal{O}$ such that the labels of which contain $l_{i}$. So $I\left(l_{i}\right)$ can be obtained by scanning the labels of all the faces in $\mathcal{O}$. This takes $O\left(K^{2}\right)$ time, since the number of faces in $\mathcal{O}$ is $O\left(K^{2}\right)$. Therefore, all $I\left(l_{i}\right)$ and $E\left(l_{i}\right)$ can be computed in $O\left(m K^{2}\right)$ time. By definition, all $I\left(v_{i}\right)$ and $E\left(v_{i}\right)$ can be computed in $O\left(n m K^{2}\right)$ time. Each of the $O(n(n+m))$ conditions in Theorem 1 can be checked in $O\left(K^{2}\right)$ time, so these conditions can be checked in $O\left(n(n+m) K^{2}\right)$ time if the overlay is computed. In conclusion, the consistency can be checked in $O((n+$ $\left.m)^{3}+n(n+m) K^{2}+m^{2} K^{2} \log K\right)$ time, where the term $(n+m)^{3}$ is the time needed to decide the path-consistency of the network.

\section{The Hybrid Consistency Problem of RCC-8}

Suppose $\Gamma$ is a complete basic RCC-8 network that involves no landmarks. Then $\Gamma$ is consistent if it is path-consistent [911]. Moreover, a solution can be constructed for each path-consistent basic network in cubic time [4]. This section shows that, however, when considering polygons, it is NP-hard to determine if a complete basic RCC-8 network involving landmarks has a solution. We achieve this by devising a polynomial reduction from $3-\mathrm{SAT}$.

In this section, for clarity, we use upper case letters $A, B, C$ (with indices) to denote landmarks, and use lower case letters $u, v, w$ (with indices) to denote spatial variables.

The NP-hardness stems from the fact that two externally connected polygons, say $A, B$, may have more than one tangential points. Assume $v$ is a spatial variable that is required to be a tangentially proper part of $A$ but externally connected to $B$. Then it is undetermined at which tangential point(s) $v$ and $B$ should meet.

Precisely, consider the configuration shown in Fig. 1)(a), where $A$ and $B$ are two externally connected landmarks, meeting at two tangential points, say $Q^{+}$and $Q^{-}$. Assume $\{u, v, w\}$ are variables that are subject to the following constraints

$$
\begin{aligned}
& u \mathbf{T P P} A, u \mathbf{E C} B, \\
& v \mathbf{T P P} B, v \mathbf{E C} A, w \mathbf{T P P} B, w \mathbf{E C} A, \\
& u \mathbf{E C} v, u \mathbf{D C} w, v \mathbf{D C} w .
\end{aligned}
$$

It is clear that $u$ is required to meet $B$ at either $Q^{+}$or $Q^{-}$, but not both (cf Fig. 1 (b,c)). The correspondence between these two configurations and the two truth values (true or false) of a propositional variable is exploited in the following reduction.

Let $\phi=\bigwedge_{k=1}^{m} \varphi_{k}$ be a 3-SAT instance over propositional variables set $\left\{p_{1}, \cdots, p_{n}\right\}$. Each clause $\varphi_{k}$ has the form $p_{r}^{*} \vee p_{s}^{*} \vee p_{t}^{*}$, where literal $p_{i}^{*}$ is either $p_{i}$ or $\neg p_{i}$ for $i=r, s, t$. We next construct a set of polygons $L$ and a complete basic RCC-8 network $\Gamma_{\phi}$, such that $\phi$ is satisfiable iff $\Gamma_{\phi}$ is satisfiable. 


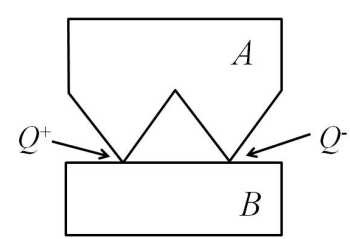

(a)

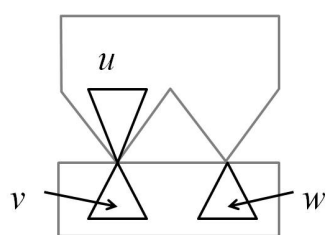

(b)

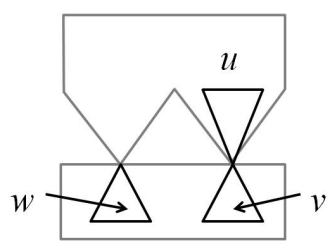

(c)

Fig. 1. Two landmarks $A, B$ that are externally connected at two tangential points $Q^{+}$and $Q^{-}$.

First, we define $A, B_{1}, B_{2}, \cdots, B_{n}$ such that for each $1 \leq i \leq n, A$ is externally connected to $B_{i}$ at two tangential points $Q_{i}^{+}$and $Q_{i}^{-}$, as shown in Fig.2

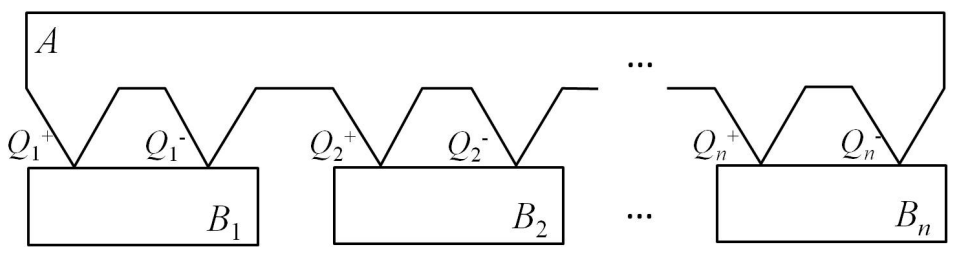

Fig. 2. Illustration of landmarks $A, B_{1}, \cdots, B_{n}$.

The variable set of $\Gamma$ is $V=\left\{u, v_{1}, \cdots, v_{n}, w_{1}, \cdots, w_{n}\right\}$. We impose the following constraints to the variables in $V$.

$$
\begin{array}{lll}
u \mathbf{T P P} A, & u \mathbf{E} \mathbf{C} B_{i}, & \\
v_{i} \mathbf{E C} A, & v_{i} \mathbf{T} \mathbf{P} B_{i}, & v_{i} \mathbf{D C} B_{j}(j \neq i), \\
w_{i} \mathbf{E} \mathbf{C} A, & w_{i} \mathbf{T P P} B_{i}, & w_{i} \mathbf{D C} B_{j}(j \neq i), \\
u \mathbf{E C} v_{i}, & u \mathbf{D C} w_{i}, & \\
v_{i} \mathbf{D C} w_{j}, & v_{i} \mathbf{D C} v_{j}(j \neq i), & w_{i} \mathbf{D C} w_{j}(j \neq i) .
\end{array}
$$

Therefore, $u$ is required to meet each $B_{i}$, at either $Q_{i}^{-}$or $Q_{i}^{+}$but not both.

For each clause $\varphi_{k}$, we introduce an additional landmark $C_{k}$, which externally connects $A$ at three tangential points, and partially overlaps $B_{i}$. The three tangential points of $C_{k}$ and $A$ are determined by the literals in $\varphi_{k}$. Precisely, suppose $\varphi_{k}=p_{r}^{*} \vee p_{s}^{*} \vee p_{t}^{*}$, then the first tangential point of $A$ and $C_{k}$ is constructed to be $Q_{r}^{+}$if $p_{r}^{*}=p_{r}$, or $Q_{r}^{-}$ if $p_{r}^{*}=\neg p_{r}$. The second and the third tangential points are selected from $\left\{Q_{s}^{+}, Q_{s}^{-}\right\}$ and $\left\{Q_{t}^{+}, Q_{t}^{-}\right\}$similarly. Take clause $p_{r} \vee \neg p_{s} \vee p_{t}$ for example, the tangential points between landmarks $C_{k}$ and $A$ should be $Q_{r}^{+}, Q_{s}^{-}$, and $Q_{t}^{+}$, as shown in Fig. 3 .

The constraints between $C_{k}$ and variables in $V$ are specified as

$$
u \mathbf{E C} C_{k}, \quad v_{i} \mathbf{P O} C_{k}, \quad w_{i} \mathbf{P O} C_{k} .
$$

Since $C_{k}$ and $A$ have three tangential points, the constraints $u \mathbf{T P P} A$ and $u \mathbf{E C} C_{k}$ imply that $u$ should occupy at least one of the three tangential points. This corresponds 


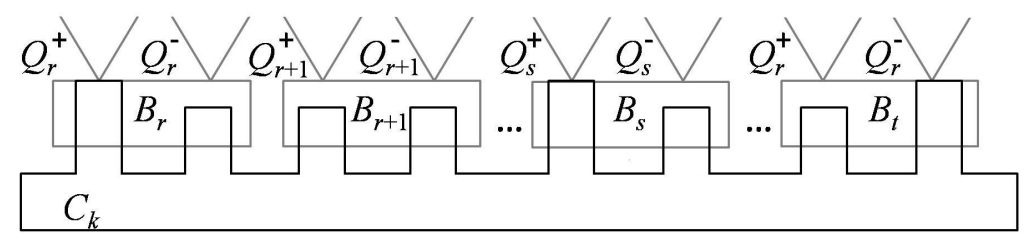

Fig. 3. Illustration of landmark $C_{k}$.

to the fact that if $\varphi_{k}$ is true under some assignment, then at least one of its three literals is assigned true.

Lemma 2. Suppose $\phi=\bigwedge_{k=1}^{m} \varphi_{k}$ is a 3-SAT instance over propositional variables set $\left\{p_{1}, p_{2}, \cdots, p_{n}\right\}$. Let $\Gamma_{\phi}$ be the basic RCC-8 network composed with constraints in (17)-(22), involving landmarks $\left\{A, B_{1}, \cdots, B_{n}, C_{1}, \cdots, C_{m}\right\}$ and spatial variables $\left\{u, v_{1}, \cdots, v_{n}, w_{1}, \cdots, w_{n}\right\}$. Then $\phi$ is satisfiable iff $\Gamma_{\phi}$ is satisfiable.

Proof. Suppose $\phi$ is satisfiable and $\pi: P \rightarrow\{$ true, false $\}$ is a truth value assignment that satisfies $\phi$. We construct regions $\bar{u}, \bar{v}_{1}, \cdots, \bar{v}_{n}, \bar{w}_{1}, \cdots, \bar{w}_{m}$ that satisfy all constraints in $\Gamma_{\phi}$.

Region $\bar{u}$ is composed of $n$ pairwise disjoint triangles in $A$. The lower vertex of the $i$-th triangle is $Q_{i}^{+}$if $\pi\left(p_{i}\right)=$ true, and $Q_{i}^{-}$otherwise. Fig. 4 shows the case that $\pi\left(p_{1}\right)=$ true, $\pi\left(p_{2}\right)=$ false, $\pi\left(p_{n}\right)=$ true.

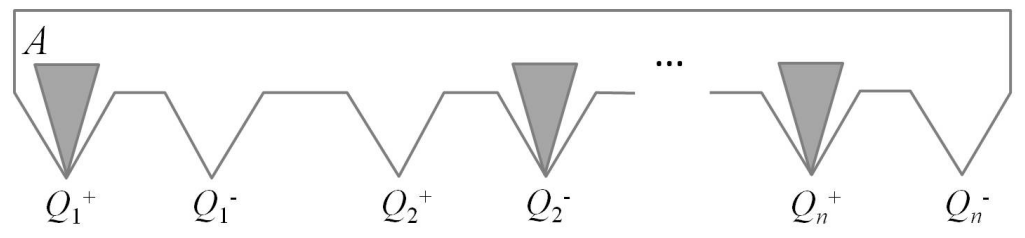

Fig. 4. Construction of variable $u$.

Regions $\bar{v}_{i}$ and $\bar{w}_{i}$ are constructed as, respectively, a triangle inside $B_{i}$. If $\pi\left(p_{i}\right)=$ true, then $Q_{i}^{+}$is a vertex of $\bar{v}_{i}$ and $Q_{i}^{-}$is a vertex of $\bar{w}_{i}$ (see Fig. 5.a)). Oppositely, if $\pi\left(p_{i}\right)=$ false, then $Q_{i}^{-}$is a vertex of $\bar{v}_{i}$ and $Q_{i}^{+}$is a vertex of $\bar{w}_{i}$ (see Fig. 5.b)). Moreover, $\bar{v}_{i}$ and $\bar{w}_{i}$ are properly chosen to make them partially overlap with each $C_{k}$.

By the construction, it is easy to see that all constraints in 17-22, except $u \mathbf{E C} C_{k}$, are satisfied. We next show $u \mathbf{E} \mathbf{C} C_{k}$ is also satisfied. That is, $\bar{u} \mathbf{E} \mathbf{C} C_{k}$. Because $\pi$ satisfies $\phi$, it also satisfies $\varphi_{k}$. That is, at least one of the three literals in $\varphi_{k}$, say $p_{r}^{*}$, is true under the assignment $\pi$. If $p_{r}^{*}=p_{r}$, then $Q_{r}^{+}$is at the boundary of $C_{k}$ by construction. In this case, we have $\pi\left(p_{r}\right)=$ true. By the construction of $\bar{u}$, we know $Q_{r}^{+}$is also at the boundary of $\bar{u}$. Similarly, if $p_{r}^{*}=\neg p_{r}$, then we can prove $Q_{r}^{-}$is a tangential point of $C_{k}$ and $\bar{u}$. Therefore, in both cases, the RCC- 8 relation between $C_{k}$ and $u$ is EC. This shows that the constructed regions $\bar{u}, \bar{v}_{1}, \cdots, \bar{v}_{n}, \bar{w}_{1}, \cdots, \bar{w}_{m}$ satisfy all constraints in $\Gamma_{\phi}$. Hence, $\Gamma_{\phi}$ is satisfiable. 


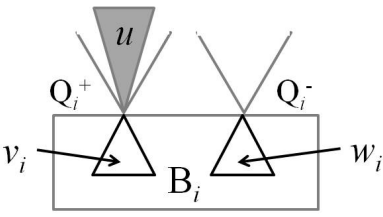

(a)

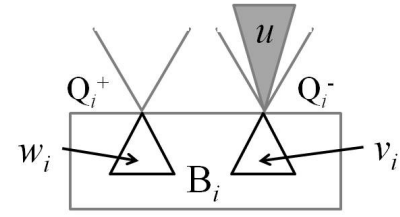

(b)

Fig. 5. Construction of variable $v_{i}$ and $w_{i} . \pi\left(p_{i}\right)=$ true (a), $\pi\left(p_{i}\right)=$ false (b).

On the other hand, suppose $\left\{\bar{u}, \bar{v}_{1}, \cdots, \bar{v}_{n}, \bar{w}_{1}, \cdots, \bar{w}_{n}\right\}$ is a solution of the network $\Gamma_{\phi}$. It is straightforward to verify that $\bar{v}_{i}$ has exactly one tangential point with $A$, namely either $Q_{i}^{-}$or $Q_{i}^{+}$. We define a truth value assignment $\pi: P \rightarrow\{$ true, false $\}$ as

$$
\pi\left(p_{i}\right)=\left\{\begin{array}{l}
\text { true, if } \bar{v}_{i} \cap A=Q_{i}^{+}, \\
\text {false, otherwise. }
\end{array}\right.
$$

We assert that $\pi\left(\varphi_{k}\right)=$ true for each $\varphi_{k}$ in $\phi$. Otherwise, suppose $\pi\left(\varphi_{k}\right)=$ false for some $\varphi_{k}=p_{r}^{*} \vee p_{s}^{*} \vee p_{t}^{*}$ in $\phi$. This only happens when $\pi\left(p_{i}^{*}\right)=$ false for $i=r, s, t$. Therefore, for $i=r, s, t$, if $p_{i}^{*}$ is positive, then by 23 , we know that $\bar{v}_{i} \cap A=Q_{i}^{-}$. Since $\bar{u} \subset A$ and $\bar{v}_{i} \mathbf{E C C} \bar{u}$, we have $Q_{i}^{-} \in \bar{u}$, which implies $Q_{i}^{+}$is not in $\bar{u}$. Similarly if $p_{i}^{*}$ is negative, then $Q_{i}^{-}$is not in $\bar{u}$. This is to say, all the three tangential points of $A$ and $C_{k}$ are not in $\bar{u}$, which contradicts with $\bar{u} \mathbf{E C} C_{k}$. Therefore, $\phi$ is satisfiable.

Corollary 1. Deciding the consistency of a complete basic RCC-8 network involving landmarks is NP-hard.

Is this problem still in NP? As long as the landmarks are polygons, the answer is yes! Recall that we write $\mathcal{O}$ for the overlay of all landmarks (cf. Theorem 3). As a subdivision, $\mathcal{O}$ consists of faces, edges and vertices. For RCC-5, only faces in $\mathcal{O}$ (i.e., the blocks) affect the consistency. For RCC-8, the vertices and the edges in $\mathcal{O}$ also need to be considered. We denote $I\left(l_{i}\right)\left(E\left(l_{i}\right), B\left(l_{i}\right)\right.$ resp.) for the set of faces, edges, and vertices contained in the interior (exterior, boundary resp.) of landmark $l_{i}$, and define $I\left(v_{i}\right)\left(E\left(v_{i}\right)\right.$ resp.) to be the set of faces, edges and vertices that are required to be in the interior (exterior resp.) of variable $v_{i}$. Each RCC- 8 constraint between a variable $v$ and a landmark $l$ is equivalent to several requirements about $I(v), E(v)$ and the boundary of $v$, with respect to $I(l), E(l)$ and $B(l)$. For example, $v \mathbf{T P P} l$ is equivalent to (i) $E(v) \supseteq E(l)$, (ii) $I(v) \subset I(l)$, and (iii) the boundary of $v$ has nonempty intersection with some edge or vertex in $B(l)$. The NP-hardness of the hybrid consistency problem of RCC-8 is mainly related to the last kind of requirement which involves the boundary of $v$, i.e., to decide whether a vertex is on the boundary of variable $v$. This can be resolved by a non-deterministic algorithm that guesses whether each vertex in $\mathcal{O}$ is on the boundary of $v$. Once the guessing is made, we can prove that, for example, either (iii) automatically holds, or it is satisfiable iff $I(v) \cup E(v) \nsubseteq B(l)$, moreover, the RCC8 constraint network can be expressed by a set of necessary conditions about $I\left(v_{i}\right)$ and $E\left(v_{i}\right)$, without involving the boundary of $v_{i}$. These conditions are also sufficient and can be checked in polynomial time. 
Theorem 4. Suppose all landmarks in a hybrid basic RCC-8 network are represented by (complex) polygons. Then deciding the consistency of a complete basic RCC-8 network involving at least one landmark is an NP-complete problem.

\section{Conclusion and Further Discussions}

In this paper, we introduced a new paradigm of consistency checking problem for qualitative calculi, which supports definitions of constraints between a constant (landmark) and a variable. Constraints like these are very popular in practical applications such as urban planning and schedule planning. Therefore, this hybrid consistency problem is more practical. Our examinations showed that for some well-behaved qualitative calculi such as PA and IA, the new hybrid consistency problem can be solved in the same way; while for some calculi e.g. RCC-5 and RCC-8, the usual composition-based reasoning approach fails to solve the hybrid consistency problem. We provided necessary and sufficient conditions for deciding if a hybrid basic RCC-5 network is consistent. Under the assumption that each landmark is represented as a polygon, these conditions can be checked in polynomial time. As for the RCC-8, we show that it is NP-complete to determine the consistency of a basic network that involves polygonal landmarks.

The hybrid consistency problem is equivalent to determining if a partial solution can be extended to a complete solution. This is usually harder than the pure consistency problem. More close connections between the pure and hybrid consistency problems are still unknown. For example, suppose the consistency problem is in NP (decidable, resp.), is the hybrid consistency problem always in NP (decidable, resp.)?

\section{References}

1. Allen, J.F.: Maintaining knowledge about temporal intervals. Commun. ACM 26(11), 832843 (1983)

2. de Berg, M., Ceong, O., van Kreveld, M., Overmars, M.: Computational Geometry : Algerithms and Applications. Springer, 3rd edn. (2008)

3. Cohn, A.G., Renz, J.: Qualitative spatial reasoning. In: van Harmelen, F., Lifschitz, V., Porter, B. (eds.) Handbook of Knowledge Representation. Elsevier (2007)

4. Li, S.: On topological consistency and realization. Constraints 11(1), 31-51 (2006)

5. Li, S., Wang, H.: RCC8 binary constraint network can be consistently extended. Artif. Intell. 170(1), 1-18 (2006)

6. Li, S., Ying, M.: Region connection calculus: Its models and composition table. Artif. Intell. 145(1-2), 121-146 (2003)

7. Ligozat, G.: Reasoning about cardinal directions. J. Vis. Lang. Comput. 9(1), 23-44 (1998)

8. Ligozat, G., Renz, J.: What is a qualitative calculus? A general framework. In: Zhang, C., Guesgen, H.W., Yeap, W.K. (eds.) PRICAI. pp. 53-64. Springer (2004)

9. Nebel, B.: Computational properties of qualitative spatial reasoning: First results. In: Wachsmuth, I., Rollinger, C.R., Brauer, W. (eds.) KI. pp. 233-244. Springer (1995)

10. Randell, D.A., Cui, Z., Cohn, A.G.: A spatial logic based on regions and connection. In: KR. pp. $165-176$ (1992)

11. Renz, J., Nebel, B.: On the complexity of qualitative spatial reasoning: A maximal tractable fragment of the region connection calculus. Artif. Intell. 108(1-2), 69-123 (1999) 